\title{
Mindfulness for Healthcare Professionals
}

\author{
Mita Mistry LicAc MBAcC MSc BA (Hons) \\ Mindfulness Based Cognitive Therapist, Acupuncturist, Columnist \\ mitamistry@ymail.com @MitaMistry
}

Cite as: Mistry M. Mindfulness for healthcare professionals. Sushruta 2019 Nov 12(1): 33

DOI: $10.38192 / 12.1 .19$

Heavy demands on health care staff include dealing with a large number of patients, long hours, restricted control over the working environment and ongoing organisational changes. Such conditions have been directly associated with growing stress levels and symptoms of burnout amongst health care professionals, and consequently, affecting the quality of care delivered to patients. ${ }^{1}$ The good news is that this is now increasingly recognised and is indeed a catalyst for change in the development of awareness aimed at building self-care skills for clinicians. In particular, there is a growing body of evidence in Mindfulness-based interventions, which have a potential role in reducing stress and burnout.

But what is mindfulness? There are many definitions of mindfulness but perhaps the most succinct and widely used, was coined by Professor Jon Kabat Zin of the University of Massachusetts Medical School. ${ }^{2}$ It is the awareness that arises through 'paying attention, on purpose, in the present moment, non-judgmentally.' This essentially translates to a way of being in the world in the 'here and now' by giving your full attention to what is happening in the current experience. Mindfulness is about cultivating a greater sense of self-awareness through building a deeper connection with our bodies and emotions, and a stronger presence within our immediate environments. You have probably experienced it before, whether you recognised it as such or not. Think of a time when you were fully engaged in an activity like writing, playing sport, reading or creating art that your entire being focused on that one activity. This heightened state of attention is mindfulness.

\section{Isn't it a bit touchy-feely?}

Indeed, there are many myths surrounding mindfulness. Some common misconceptions are; it is a way of blanking your mind or going into a trance or even that it is controlling your thoughts to think positively and merely a relaxation technique rooted in ancient religious beliefs or esoteric spirituality. Whilst the origins of mindfulness stem from Buddhist and Hindu meditation, the practice itself is completely secular, it is essentially teaching life skills for coping with the human experience which has gained respect and credibility in therapeutic terms. Over the past twenty years mindfulness has been the subject of more controlled clinical research ${ }^{3}$. It has been proven with scientific rigour in the treatment of a number of 
psychological and physical conditions. It can help manage a number of mental health disorders, including anxiety and depression.

The Oxford Centre for Mindfulness has found that Mindfulness-Based Cognitive Therapy (MBCT) prevents depression relapse in recurrent depression. The National Institute for Health and Clinical Excellence (NICE) has recommended MBCT in their Guidelines for Management of Depression $(2004,2009){ }^{4}$ for service users who have had three or more episodes of depression. Other research shows significant improvement in burnout scores and mental well- being for a broad range of healthcare providers using mindfulness-based stress reduction education. And it can help achieve a sense of calm in our overloaded daily lives. By increasing self-awareness particularly of one's stress levels through understanding one's own emotional and psychological triggers creates a pathway to be responsive to situations rather than reactive. Greater self-awareness also helps to increase ability to reflect and subsequently take proactive measures to improve one's boundaries in the workplace and resolve conflicts as well as attending to others including yourself and service users with more compassion. And let's face it we are often good at extending compassion for others, but not so much for ourselves.

Where mindfulness can feel like self-care, self-compassion can often be mixed up with feelings of self-indulgence ${ }^{5}$ and therefore can be overlooked, yet it is crucial for clinicians. But there is a growing interest in mindfulness for self-compassion especially in a healthcare setting where clinicians can be vulnerable to stress overload and compassion fatigue owing to the emotionally exhausting environment ${ }^{6}$. Having compassion for others requires having compassion for oneself and a common sense of humanity. Beddoe \& Murphy $\underline{7}$ found that nurses who participated in a Mindfulness-Based Stress Reduction program reported that their mindfulness practice helped them to develop more compassion and empathy for their patients, and also helped their own self-compassion so they didn't take on the negative emotions of their patients. In another study, Shapiro et al ${ }^{8}$ also found that health care professionals who completed a mindfulness program reported an increase in feelings of selfcompassion and reduced stress. Both mindfulness and self-compassion involve promoting an attitude of curiosity and non-judgment towards one's experiences.

Research suggests that mindfulness interventions, particularly those with a focus on compassion has the potential to increase self-compassion among health care workers, which in turn, shows promising results for reducing stress and increasing the effectiveness of clinical care. ${ }^{9}$ Whilst mindfulness may not be a fix to "cure all" for everyone, it is a way of meeting our experience with the presence of mind to respond skilfully to life's challenges, rather than reacting based on intense emotions. And with growing stress levels and burnout amongst health professionals, and increasing evidence that they could benefit from mindfulness interventions what is there to lose? 


\section{References}

1. Montgomery A, Panagopoulou E, Esmail A, Richards T, Maslach C. Burnout in healthcare: the case for organisational change BMJ 2019; 366 :14774

2. https://umassmed.edu/cfm/About-Us/people/2-Meet-Our-Faculty/Kabat-ZinnProfile/

3. Ospina $\mathrm{MB}$, Bond $\mathrm{K}$, Karkhaneh $\mathrm{M}$, Tjosvold $\mathrm{L}$, Vandermeer $\mathrm{B}$, Liang $\mathrm{Y}$, Bialy $\mathrm{L}$, Hooton $\mathrm{N}$, Buscemi N, Dryden DM, Klassen TP. Meditation practices for health: state of the research. Evid Rep Technol Assess (Full Rep). 2007 Jun;(155):1-263.

4. https://www.nice.org.uk/guidance/CG90

5. Susan L. Woods, Patricia Rockman, and Evan Collins. Mindfulness-Based Cognitive Therapy. 2016 American Mindfulness Research Association.

6. Neff KD. The Role of Self-Compassion in Development: A Healthier Way to Relate to Oneself. Hum Dev. 2009 Jun;52(4):211-214.

7. Beddoe, A.E. and Murphy, S.O. (2004) Does Mindfulness Decrease Stress and Foster Empathy among Nursing Students? Journal of Nursing Education, 43, 305-312.

8. Cohen-Katz J ${ }^{1}$, Wiley SD, Capuano T, Baker DM, Shapiro S. The effects of mindfulness-based stress reduction on nurse stress and burnout: a quantitative and qualitative study. Holist Nurs Pract. 2004 Nov-Dec;18(6):302-8.

9. Raab K $\mathrm{K}^{1}$. Mindfulness, self-compassion, and empathy among health care professionals: a review of the literature. J Health Care Chaplain. 2014;20(3):95-108. 